\title{
COMMUTATORS IN A SEMI-SIMPLE LIE GROUP
}

SAMUEL PASIENCIER AND HSIEN-CHUNG WANG ${ }^{1}$

1. Introduction. K. Shoda [3] has proved that each element of the special linear group $S L(n, K)$ over an infinite and algebraically closed field $K$ is a commutator. This result has been generalized by $\mathrm{R}$. C. Thompson [4] for arbitrary $K$ when $n \neq 2$. For a complex semi-simple Lie group ${ }^{2} G, \mathrm{M}$. Goto [2] showed that the complement $G \backslash C$ of the set $C$ of commutators is at most $(2 n-2)$-dimensional where $2 n$ denotes the topological dimension of $G .^{3}$ It is the aim of this paper to prove that $G$ and $C$ actually coincide. In the discussions, the Weyl group of $G$ plays an important rôle.

2. The theorem. Let $G$ be a Lie group and $G$ its Lie algebra. We shall always use Ad. and ad. to denote the linear adjoint representations of $G$ and $G$ on $G$. An element $x$ of $G$ is called semi-simple if all the invariant subspaces of Ad. $x$ are simple; and is called unipotent if $I$-Ad. $x$ is nilpotent, where $I$ denotes the identity endomorphism of $G$. By a regular element of $G$, we mean an element $x$ such that the multiplicity of one as a root of the characteristic equation of Ad. $x$ is minimal. Nonregular elements are called singular. Semi-simple, regular and singular elements of the Lie algebra $G$ are defined by using ad. in the same manner.

Now, let us confine our attention to a complex, semi-simple Lie group $G$. Its complex Lie algebra will be denoted by $G$. Take a Cartan subalgebra $H$ of $G$ and choose a basis $h_{1}, \cdots, h_{l}$ of $H$ such that, for each root $\alpha$ of $G, \alpha\left(h_{i}\right)$ assumes rational values, $i=1, \cdots, l$. An ordering of the roots of $G$ may be defined by means of this base. The totality of the root vectors $e_{\alpha}$ with $\alpha>0$ spans a nilpotent subalgebra $\boldsymbol{N}$ of $\boldsymbol{G}$. The sum $\boldsymbol{M}=\boldsymbol{H}+\boldsymbol{N}$ is a maximal solvable subalgebra of $\boldsymbol{G}$. Let $M, H, N$ be the subgroups generated by $M, H, N$ respectively in $G$. All of them are closed. We can see easily that $M=H \cdot N$ and that $H \cap N$ contains only the identity. In fact, $H$ is a Cartan subgroup and $N$ is the identity component of the set of all unipotent elements in $M$. Moreover $N$ is normal in $M$.

The proof of our main result depends on the following two lemmas.

Presented to the Society, September 1, 1960 under the title Commutators in a complex semi-simple Lie group; received by the editors August 22, 1960 and, in revised form, November 3,1961 .

1 Supported in part by the National Science Foundation.

2 Lie groups are always assumed to be connected.

3 Goto proved that each semi-simple element is a commutator. 
Lemma 1. Let $x \in M, x=s n, s \in H, n \in N$. If $s$ is a regular element of $G$, then $x$ and s are conjugate in $M$.

LemMa 2. For any $s \in H$, there exists a regular element $r \in H$, and an element $g$ of $G$, such that

$$
g H g^{-1}=H, \quad g r g^{-1}=r s .
$$

Let us now establish our theorem, assuming these two lemmas.

Suppose $G$ to be a connected, complex semi-simple Lie group and $M, H, N$ have the same meaning as above. Each element of $G$ is conjugate to an element of $M$, and, therefore, it suffices to show that any $x \in M$ is a commutator in $G$. Let us write $x=s n$ with $s \in H$ and $n \in N$. By Lemma 2, there exists a $g \in G$ and $r \in H$ such that $g r g^{-1}=r s$, and $r$ is regular. Hence $s r \in H$ and $s r$ is regular. From Lemma 1, we can find $b \in M$ satisfying the equality

$$
b s r b^{-1}=s r n
$$

and thus

$$
x=s n=r^{-1} b s r b^{-1}=r^{-1} b g r g^{-1} b^{-1}=r^{-1}(b g) r(b g)^{-1} .
$$

Therefore, if Lemmas 1, 2 are valid, we have proved the

TheOREM. In a complex semi-simple Lie group, each element is a commutator.

3. Proof of Lemma 1. Suppose $G, H, N, M$ have the same meaning as above. We consider the linear adjoint representation Ad. of $G$ on $G$. There exists a basis of $G$ referred to which each endomorphism in Ad. $N$ takes the form of a right triangular matrix, and those in Ad. $H$ diagonal matrices. Since $n \in N$ is unipotent in $G$, the matrix Ad. $n$ has all diagonal entries equal to unity. Hence Ad. $x$ and Ad.s are two right triangular matrices with the same set of diagonal elements and hence the same set of characteristic roots. Since by hypothesis $s$ is regular in $G$, so is $x$. We know that $G$ and $M$ have the same rank. It follows that $x$ is also a regular element of $M$. There exists then an element $y$ of $M$ such that

$$
y^{-1} x y=y^{-1} \text { sny } \in H,
$$

because $H$ is a Cartan subgroup not only of $G$, but also of $M$. Writing $y=u v, u \in H, v \in N$ and taking account of the commutativity $u s=s u$, we have

$$
\left(s^{-1} v^{-1} s\right)\left(u^{-1} n u\right) v=s^{-1}\left(y^{-1} x y\right) \in s^{-1} H=H .
$$


But $v, u$ are elements of $N$, and $N$ is normal in $M$, and so

$$
s^{-1} v^{-1} s u^{-1} n u v \in N \text {. }
$$

From the fact that $H \cap N=e$, it follows that

$$
s^{-1} v^{-1} s u^{-1} n u v=e
$$

whence

$$
y^{-1} x y=s
$$

Lemma 1 is thus proved.

4. Weyl group. In the proof of Lemma 2, we have to use extensively the Weyl group $W$ of a complex, semi-simple Lie group. Let us, in this section, introduce some notations and give the properties of $W$ relevant to our discussion.

Let $H$ be a Cartan subgroup of a complex, semi-simple Lie group $G$, and $\boldsymbol{H}$ the corresponding complex Lie algebra. We know that $\boldsymbol{H}$ is a complex vector space of dimension $n$ where $n$ is the rank of $G$. Let $B$ denote the restriction of the Killing form of $G$ on $H$. To each root $\alpha$ of $G$, there corresponds a vector $h_{\alpha}$ in $\boldsymbol{H}$ defined by $B\left(h_{\alpha}, h\right)$ $=\alpha(h), h \in H$. For simplicity we shall call $h_{\alpha}$ the root normal corresponding to $\alpha$.

Let $\epsilon: H \rightarrow H$ be the exponential map. All the elements $h$ of $\boldsymbol{H}$ such that $\epsilon(h)$ belong to the center of $G$ form a lattice $\mathfrak{B}$ in $H$. This $\mathfrak{B}$ has $\boldsymbol{n}$ independent generators. Since Ad. $\epsilon(h)=\exp (\operatorname{ad} . h), h \in \boldsymbol{H}$, we see immediately that $\epsilon(h)$ is regular if and only if, for each root $\alpha$ and each $K \in \mathfrak{B}, \alpha(h+K) \neq 0$.

The normalizer of $H$ in $G$ is finite over $H$, and thus induces a finite group $W$ of automorphisms of $H$. This group is called the Weyl group. Passing to differentials, we can also regard $W$ as a group of automorphisms of the Lie algebra $\boldsymbol{H}$. We shall use the same notation $W$ in both cases. We note that for each $\sigma \in W$, the diagram

$$
\begin{gathered}
H \stackrel{\sigma}{\rightarrow} H \\
\epsilon \uparrow \quad \text { }{ }^{\uparrow} \epsilon \\
H \underset{\sigma}{\rightarrow} H
\end{gathered}
$$

is commutative. Let $\alpha$ be a root. With respect to the quadratic form $B$, there corresponds an involution of $\boldsymbol{H}$ :

$$
S_{\alpha}: h \rightarrow h-2 \frac{B\left(h, h_{\alpha}\right)}{B\left(h_{\alpha}, h_{\alpha}\right)} h_{\alpha}
$$


which we shall call the reflection about $\alpha$. The Weyl group $W$ is generated by these reflections.

Let $\alpha_{1}, \alpha_{2}, \cdots, \alpha_{t}$ be $t$ roots of $G, S_{i}$ the reflection about $\alpha_{i}, h_{i}$ the root normal corresponding to $\alpha_{i}$, and $\sigma=S_{1} \cdot S_{2} \cdots \cdot S_{t}$. Then the image $(\sigma-I)(H)$ is contained in the linear space $P$ spanned by $h_{1}, h_{2}, \cdots, h_{t}$. If, moreover, the $\alpha$ 's are linearly independent, then $(\sigma-I)(H)$ actually coincides with $P$. All these can be seen by using induction on $t$ (cf. [5]).

5. A theorem of Kostant. Before proving Lemma 2, let us first establish the following unpublished result of B. Kostant:

$(5.1)^{4}$ Let $\sigma$ be an element of the Weyl group $W$, and I the identity automorphism of the Cartan subalgebra $\boldsymbol{H}$. (A) For any root normal $h_{\phi} \in(\sigma-I)(H)$, the dimension of the image of $\sigma S_{\phi}-I$ is lower than that of $\sigma-I$. (B) There exist linearly independent root normals $h_{\alpha_{1}}, h_{\alpha_{2}}, \cdots$, $h_{\alpha_{t}}$ such that $t=\operatorname{dim}(\sigma-I)(H)$ and

$$
\sigma=S_{\alpha_{1}} \cdot S_{\alpha_{2}} \cdots S_{\alpha_{t}} .
$$

Proof. Denote by $H_{1}$ the space of fixed points of $\sigma$, and $H_{2}$ the orthogonal complement of $H_{1}$ in $H$. Then $H=H_{1} \oplus H_{2}$ and

$$
h_{\phi} \in H_{2}=(\sigma-I)(H)=\left(\sigma^{-1}-I\right)(H)
$$

on account of the semi-simplicity of $\sigma$. It is evident that $B\left(h_{\phi}, H_{1}\right)=0$. Therefore, for each $h \in H_{1}, \sigma S_{\phi}(h)=\sigma(h)=h$, or in other words,

$$
\operatorname{ker}(\sigma-I)=H_{1} \subset \operatorname{ker}\left(\sigma S_{\phi}-I\right) .
$$

From (5.2), there exists $k \in H_{2}, k \neq 0$, with $\left(\sigma^{-1}-I\right) k=h_{\phi}$ whence $\sigma^{-1}(k)=k+h_{\phi}$. We have then $B(k, k)=B\left(\sigma^{-1}(k), \sigma^{-1}(k)\right)$ $=B\left(k+h_{\phi}, k+h_{\phi}\right)$. It follows that $B\left(h_{\phi}, h_{\phi}\right)+2 B\left(k, h_{\phi}\right)=0$, and then

$$
\sigma S_{\phi}(k)=\sigma\left(k-\frac{2 B\left(k, h_{\phi}\right)}{B\left(h_{\phi}, h_{\phi}\right)} h_{\phi}\right)=\sigma\left(k+h_{\phi}\right)=k .
$$

This together with (5.3) tells us that $\operatorname{ker}\left(\sigma S_{\phi}-I\right)$ is of higher dimension than that of ker $(\sigma-I)$. Part (A) is thus proved.

To prove (B), let us use induction on the dimension $n(\sigma)$ of $(\sigma-I)(H)$. When $n(\sigma)=0$, this is trivally true. Now assume $n(\sigma)$ $=t>0$ and assume the proposition (5.1) to be valid for $n(\sigma)<t$. Consider the decomposition $H=H_{1} \oplus H_{2}$ as defined above. Since $\sigma$ is not the identity, $\boldsymbol{H}_{1}$ cannot contain any regular element. To each $h$ of $H_{1}$, there exists then a root normal $h_{\alpha}$ with $B\left(h_{\alpha}, h\right)=0$. But we have only a finite number of root normals and so there must be a root

- This proposition and its proof are due to B. Kostant. 
normal $h_{\beta}$ satisfying $B\left(h_{\beta}, H_{1}\right)=0$, or what is the same, $h_{\beta} \in H_{2}$. From Part (A),

$$
\operatorname{dim}\left(\left(\sigma S_{\beta}-I\right)(H)\right)<\operatorname{dim}((\sigma-I)(H))=t .
$$

By induction hypothesis, we can find roots $\alpha_{1}, \alpha_{2}, \cdots, \alpha_{u}$ such that $\sigma S_{\beta}=S_{\alpha_{1}} \cdot S_{\alpha_{2}} \cdots \cdots S_{\alpha_{u}}$ where $u=\operatorname{dim}\left(\left(\sigma S_{\beta}-I\right)(\boldsymbol{H})\right)$. Hence $\sigma=S_{\alpha_{1}} \cdots S_{\alpha_{u}} \cdot S_{\beta}$. Since $(\sigma-I(H)$ is contained in the linear space spanned by $h_{\alpha_{1}}, \cdots, h_{\alpha_{u}}$ and $h_{\beta}, t$ is not greater than $u+1$. Inequality (5.4) then implies that $t=u+1$ and that $h_{\alpha_{1}}, \cdots, h_{\alpha_{u}}, h_{\beta}$ are linearly independent. (5.1) is proved.

REMARK. From (5.1) and the last paragraph of $\$ 4$, we know that, for each element $\sigma$ of the Weyl group, $(\sigma-I)(H)$ is spanned by root normals.

(5.5) There exists an element $\theta$ of $W$ such that (i) $\theta-I$ is one-to-one, and (ii) to each singular element $h \in H,(\theta-I)(h)$ is a linear combination of at most $n-1$ root normals where $n$ is the rank of $G$.

Proof. Take any $n$ linearly independent roots $\alpha_{1}, \alpha_{2}, \cdots, \alpha_{n}$ and put $\theta=S_{\alpha_{1}} S_{\alpha_{2}} \cdots S_{\alpha_{n}}$. Then $\theta-I$ is one-to-one. Let $h$ be a singular element in $\boldsymbol{H}$. Take a root $\phi$ with $\phi(h)=0$. Then $S_{\phi}(h)=h$ and $(\theta-I)(h)$ $=\left(\theta S_{\phi}-I\right)(h)$. By (5.1), $\left(\theta S_{\phi}-I\right)(H)$ is at most $(n-1)$-dimensional, and by the above remark, $\left(\theta S_{\phi}-I\right)(H)$ is spanned by root normals. Hence $(\theta-I)(h)$ is a linear combination of $n-1$ root normals. (5.5) is thus proved.

The authors originally proved (5.5) by using classification of simple Lie algebras. The simpler proof given above was suggested by $B$. Kostant to whom the authors wish to express their thanks.

6. Proof of Lemma 2. Let us recall that $G$ is a complex semi-simple Lie group of rank $n, H$ a Cartan subgroup of $G$, and $G, H$ are the Lie algebras of $G$ and $H$, respectively. Suppose $s$ to be an element of $H$. We want to show the existence of a regular element $r$ in $H$ and an element $\sigma$ of the Weyl group $W$ such that $\sigma(r)=r s$.

Let us use induction on the rank of $G$. When $n=0$, this becomes trivial. Now assume Lemma 2 to be valid for groups of rank less than $n$. Since the exponential map $\epsilon: H \rightarrow H$ is surjective, we can find an $h_{0} \in H$ such that $\epsilon\left(h_{0}\right)=s$. Choose an element $\theta$ of the Weyl group having the two properties mentioned in (5.5). There exists then an $h_{1} \in H$ with $(\theta-I) h_{1}=h_{0}$. Suppose that $\epsilon\left(h_{1}\right)$ is regular. Then

$$
\theta\left(\epsilon\left(h_{1}\right)\right)=\epsilon\left(\theta\left(h_{1}\right)\right)=\epsilon\left(h_{0}+h_{1}\right)=\epsilon\left(h_{0}\right) \epsilon\left(h_{1}\right)=s \cdot \epsilon\left(h_{1}\right)
$$

and Lemma 2 is thus proved.

Now suppose that $\epsilon\left(h_{1}\right)$ is not regular. Then there exists a root $\alpha$ of $G$ and an element $l \in \mathfrak{B}$ such that $\alpha\left(h_{1}+l\right)=0$. By our choice of $\theta$, 
the vector $h_{0}^{\prime}=(\theta-I)\left(h_{1}+l\right)$ is a linear combination of root normals $h_{\beta_{1}}, \cdots, h_{\beta_{n-1}} .{ }^{5}$ Let $\Sigma$ be the totality of roots of $G$ which depend linearly on $\beta_{1}, \cdots, \beta_{n-1}$, and let $H^{\prime}$ be the linear subspace spanned by $\left\{h_{\beta}: \beta \in \Sigma\right\}$ and $G^{\prime}$ that spanned by $\left\{h_{\beta}, e_{\beta}: \beta \in \Sigma\right\}$ where $e_{\beta}$ denotes the root vector corresponding to the $\operatorname{root} \beta$. We see immediately that $G^{\prime}$ is semi-simple and of rank $n-1$, with $H^{\prime}$ as one of its Cartan subalgebras. Denote by $G^{\prime}, H^{\prime}$ the subgroups of $G$ generated by $G^{\prime}$, $H^{\prime}$, respectively. Since $G^{\prime}$ is semi-simple, $G^{\prime}$ is closed in $G$ and hence a Lie group. Now let us see that $s \in G^{\prime}$. In fact, we have

$$
s=\epsilon\left(h_{0}\right)=\epsilon\left((\theta-I) h_{1}\right)=\epsilon\left(h_{0}^{\prime}-(\theta-I) l\right)=\epsilon\left(h_{0}^{\prime}\right) \epsilon((I-\theta) l) .
$$

But $l \in \mathfrak{B}, \epsilon(l)$ belongs to the center of $G$, and therefore $\theta(\epsilon(l))=\epsilon(l)$, whence

$$
\epsilon((\theta-I) l)=\epsilon(\theta(l)) \epsilon(-l)=\theta(\epsilon(l)) \cdot(\epsilon(-l))=e .
$$

It follows then

$$
s=\epsilon\left(h_{0}^{\prime}\right) \in \epsilon\left(H^{\prime}\right)=H^{\prime} \subset G^{\prime} .
$$

The semi-simple group $G^{\prime}$ being of rank $n-1$, we know from the induction hypothesis that there exists an element $\sigma$ of the Weyl group $W^{\prime}$ of $G^{\prime}$, and an element $r^{\prime}$ in $H^{\prime}$ which is regular in $G^{\prime}$ such that

$$
\sigma\left(r^{\prime}\right)=s r^{\prime} .
$$

Since $H^{\prime} \subset H$ and each root of $G^{\prime}$ is obtained from a root of $G$ by restriction, the Weyl group $W^{\prime}$ is imbedded in the Weyl group $W$ in a natural manner. We can therefore regard $\sigma$ to be an element of $W$. However, the element $r^{\prime}$, though regular in $G^{\prime}$, is not necessarily regular in $G$. Now let us modify it to suit our purpose. Take an element $h^{\prime} \in H^{\prime}$ such that $\epsilon\left(h^{\prime}\right)=r^{\prime}$, and take a nonzero vector $j$ in $\boldsymbol{H}$ orthogonal to $H^{\prime}$. Then $j$ and $H^{\prime}$ span $H$. Moreover,. $(\sigma-I) j=0, \beta(j)=0$, $\beta \in \Sigma$. Let $\alpha_{1}, \cdots, \alpha_{s}$ be the totality of roots of $G$ which do not depend linearly on $\beta_{1}, \cdots, \beta_{n-1}$. Then $\alpha_{m}(j) \neq 0$ and then there exists a complex number $c$ such that

$$
\alpha_{m}\left(h^{\prime}+c j\right) \not \equiv 0(\bmod 2 \pi i),
$$$$
m=1,2, \cdots, s,
$$

or what is the same

$$
\alpha_{m}\left(h^{\prime}+c j+k\right) \neq 0, \quad k \in \mathfrak{B} .
$$

Since $r^{\prime}$ is regular in $G^{\prime}$, we have

$$
\beta\left(h^{\prime}+c j\right)=\beta\left(h^{\prime}\right) \not \equiv 0(\bmod 2 \pi i), \quad \beta \in \Sigma .
$$

- Without loss of generality, we can assume these $n-1$ root normals to be linearly independent. 
Let $r=\epsilon\left(h^{\prime}+c j\right)$, then $r=\epsilon\left(h^{\prime}\right) \cdot \epsilon(c j)=r^{\prime} \epsilon(c j)$, and from (5.2), we have

$$
\begin{aligned}
\sigma(r) & =\sigma\left(r^{\prime}\right) \cdot \sigma(\epsilon(c j))=\sigma\left(r^{\prime}\right) \cdot \epsilon(\sigma(c j)) \\
& =\sigma\left(r^{\prime}\right) \epsilon(c j)=s r^{\prime} \cdot \epsilon(c j)=s r .
\end{aligned}
$$

Moreover, (6.3) and (6.4) tell us that $r$ is regular in G. Lemma 2 is thus proved.

\title{
REFERENCES
}

1. E. Cartan, La géometrie des groupes simples, Ann. Mat. Pura Appl. 4 (1927), 209-256.

2. M. Goto, $A$ theorem on compact semi-simple groups, J. Math. Soc. Japan 1 (1948), 270-273.

3. K. Shoda, Einige Satze ilber Matrizen, Japan J. Math. 13 (1937, 361-365).

4. R. C. Thompson, Commutators of matrices. III, Abstracts 565-16, Notices Amer. Math. Soc. 7 (1960), 194.

5. B. Kostant, The principal 3-dimensional subgroup and the Betti numbers of a complex simple Lie group, Amer. J. Math. 81 (1959), 973-1032.

NORTHWESTERN UNIVERSITY

\section{SOME INTEGRAL FORMULAS FOR THE COMPLETE ELLIPTIC INTEGRALS OF THE FIRST AND SECOND KIND}

\author{
L. CARLITZ ${ }^{1}$
}

In the usual notation let

$$
\begin{aligned}
& K(k)=\frac{\pi}{2} \sum_{0}^{\infty} \frac{\left(\frac{1}{2}\right)_{n}\left(\frac{1}{2}\right)_{n}}{n ! n !} k^{2 n} \\
& E(k)=\frac{\pi}{2} \sum_{0}^{\infty} \frac{\left(-\frac{1}{2}\right)_{n}\left(\frac{1}{2}\right)_{n}}{n ! n !} k^{2 n}
\end{aligned}
$$

denote the complete elliptic integrals of the first and second kind, respectively. It is easily verified that (2) implies

Received by the editors September 26, 1961 and, in revised form, November 21, 1961.

${ }^{1}$ Supported in part by National Science Foundation grant G-16485. 